\title{
Vagus Nerve Stimulation for Treatment of Inflammation: Systematic Review of Animal Models and Clinical Studies
}

\author{
Harwood Kwan, ${ }^{1}$ Luca Garzoni, ${ }^{2}$ Hai Lun Liu, ${ }^{1}$ Mingju Cao, ${ }^{1}$ Andre Desrochers, ${ }^{3}$ Gilles Fecteau, ${ }^{3}$ \\ Patrick Burns, ${ }^{3}$ and Martin G Frasch ${ }^{1,4,5}$
}

${ }^{1}$ Department of Obstetrics and Gynecology and Department of Neurosciences, University of Montreal, Montreal, Quebec, Canada; ${ }^{2}$ Department of Pediatrics, CHU Ste-Justine Research Centre, University of Montreal, Montreal, Quebec, Canada; ${ }^{3}$ Department of Clinical Sciences, University of Montreal, St-Hyacinthe, Quebec, Canada; ${ }^{4}$ Centre de Recherche en Reproduction Animale, Faculty of Veterinary Medicine, University of Montreal, St-Hyacinthe, Quebec, Canada; and ${ }^{5}$ Department of Obstetrics and Gynecology, University of Washington, Seattle, Washington, United States of America

\begin{abstract}
Vagus nerve stimulation (VNS) has been used since 1997 for treatment of drug-resistant epilepsy. More recently, an off-label use of VNS has been explored in animal models and clinical trials for treatment of a number of conditions involving the innate immune system. The underlying premise has been the notion of the cholinergic antiinflammatory pathway (CAP), mediated by the vagus nerves. While the macroanatomic substrate - the vagus nerve - is understood, the physiology of the pleiotropic VNS effects and the "language" of the vagus nerve, mediated brain-body communication, remain an enigma. Tackling this kind of enigma is precisely the challenge for and promise of bioelectronic medicine. We review the state of the art of this emerging field as it pertains to developing strategies for use of the endogenous CAP to treat inflammation and infection in various animal models and human clinical trials. This is a systematic PubMed review for the MeSH terms "vagus nerve stimulation AND inflammation." We report the diverse profile of currently used VNS antiinflammatory strategies in animal studies and human clinical trials. This review provides a foundation and calls for devising systematic and comparable VNS strategies in animal and human studies for treatment of inflammation. We discuss species-specific differences in the molecular genetics of cholinergic signaling as a framework to understand the divergence in VNS effects between species. Brain-mapping initiatives are needed to decode vagus-carried brain-body communication before hypothesis-driven treatment approaches can be devised.
\end{abstract}

Online address: www.bioelecmed.org

doi: 10.15424/bioelectronmed.2016.00005

\section{INTRODUCTION}

\section{Rationale}

Vagus nerve stimulation (VNS) has been used for treatment of drug-resistant epilepsy since 1997, when the US Food and Drug Administration approved it (1-3). More recently, an off-label use of this well-tolerated treatment modality has been explored in multiple animal experimental models and clinical trials for treatment of a number of conditions involving the innate immune system $(4,5)$. The underlying systemic antiinflammatory mechanism is mediated by the vagus nerves relaying onto the spleen's $\alpha 7$ nicotinic acetylcholine receptor $(\alpha 7 \mathrm{nAChR})$ expressing macrophages as part of the cholinergic antiinflammatory pathway (CAP) (6). While the macroanatomic wiring through the vagus nerve seems clear, the physiology of the pleiotropic VNS effects and the "language" of the vagus nerve-mediated brain-body

Address correspondence to Martin $G$ Frasch, Department of Obstetrics and Gynecology, University of Washington, 1959 NE Pacific Street, Box 356460, Seattle, WA 98195.

Phone: 206-543-5892; Fax: 206-543-3915; E-mail: mfrasch@uw.edu.

Submitted July 11, 2016; Accepted for Publication August 23, 2016; Published Online

(www.biolecmed.org) September 14, 2016.

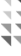

Feinstein Institute
for Medical Research

Northwell Health 
Eligibility Criteria, Information Sources and Search Strategy

We included any studies listed on PubMed in the English language that met the search term criteria (vagus nerve stimulation [MeSH terms] AND inflammation [MeSH terms]). All years up to June 27, 2016, were considered. The results are depicted in Figure 1 (PRISMA flow diagram). One article was found through Google Scholar when searching for a full-text version of another paper, Shi et al., Effects of efferent vagus nerve excitation on inflammatory response in heart tissue in rats with endotoxemia (article in Chinese).

Study selection. All study designs were considered.

Data collection process and data items. We extracted data on animal model used, location and site of VNS, frequency, intensity, pulse and stimulation durations.

Risk of bias in individual studies. Our inclusion criteria were very broad. As such, while the review has high precision, there is also potential for bias from combining studies in various animal and human trials with acute and chronic experimental designs and somewhat variable readouts. Aside from actual VNS settings, its effects on inflammation may vary by species, gender, age, anatomical site, duration of treatment application and time horizon of readouts (acute versus chronic).

Summary measures. We addressed the risk of bias by not only providing the VNS settings used throughout the literature, but also organizing the results by animal species, including gender whenever possible, VNS treatment duration and resulting effect on inflammation.

Synthesis of results. All PubMed hits were imported into EndNote software and reviewed based on the following criteria: animal model, gender, age, VNS site and duration, frequency, amplitude, intensity and duration of the impulse. If the study was conducted during a single day $(<24 \mathrm{~h})$, it was noted as acute; otherwise it was deemed chronic. Lastly, the outcome of the VNS was noted as decreasing or increasing inflammation. All data have been summarized in Table 1 .

\section{RESULTS}

Study selection and characteristics are summarized in Figure 1 (PRISMA flow diagram).

We identified 290 records, of which 36 were deemed eligible and reviewed.

All studies were conducted in adult subjects (Table 1). In total, $80 \%$ of the studies were conducted in rodents, 19 in rats and nine in mice; $69 \%$ of the studies were done in male adult subjects, and left or right cervical vagus nerve was stimulated. We found a large variance in VNS settings, with approximately one-third of the studies not reporting the intensity of the stimulus. A total of $77 \%$ of the studies were designed as acute protocols (less than $24 \mathrm{~h}$ VNS treatment) and observed the inflammatory profile for less than $24 \mathrm{~h}$. All rodent VNS studies except for one resulted in reduction of inflammation (8). Meanwhile, two of the three human studies did not show an antiinflammatory effect $(9,10)$, while one, a recent study by Koopman et al., demonstrated in vitro antiinflammatory programming effects in peripheral blood monocytes due to VNS in adult human subjects with no known immune system dysfunction and in patients with diagnosed rheumatoid arthritis (42). In the latter, chronic improvement in disease

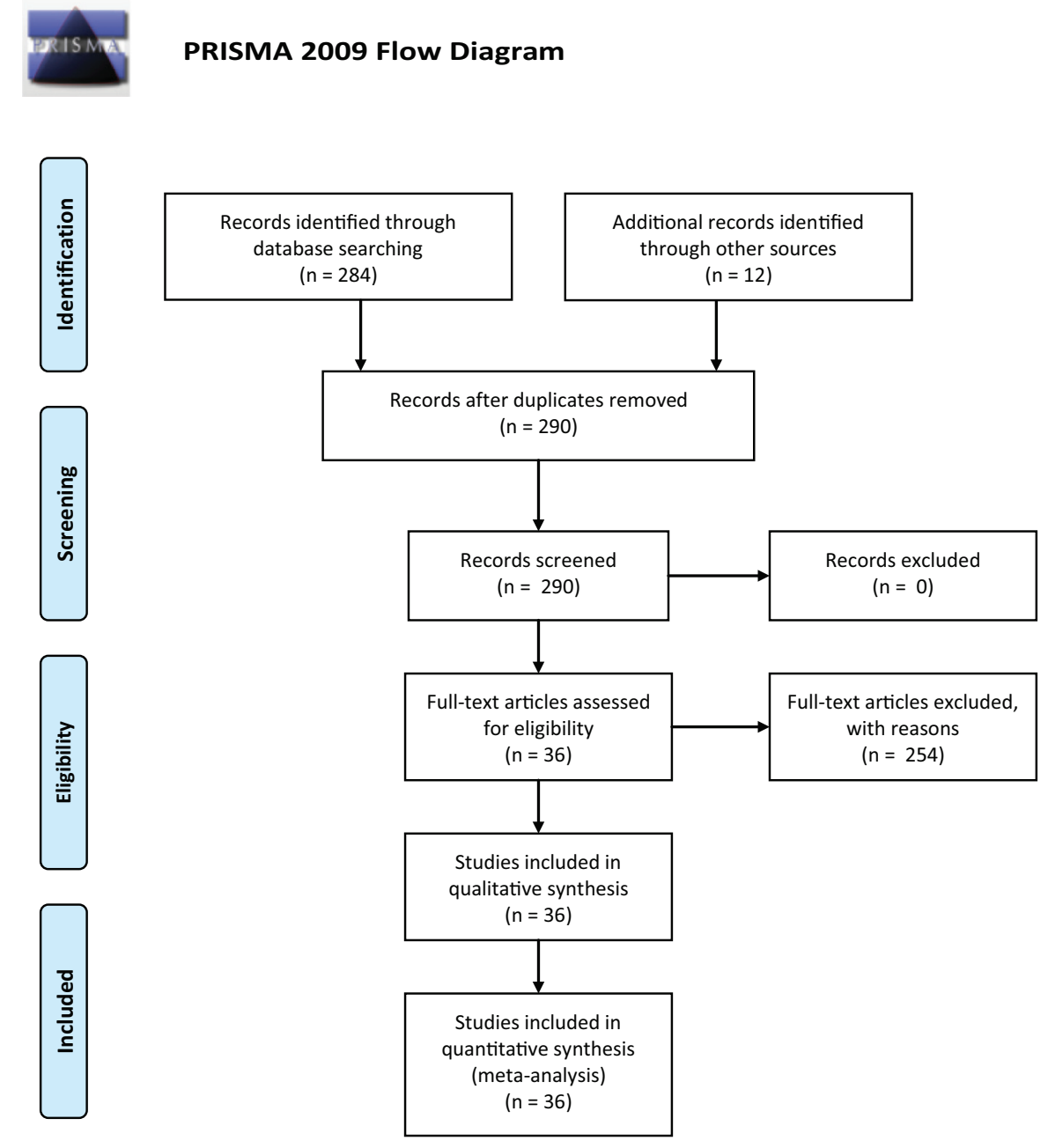

Figure 1. Approach to systematic review of studies on vagus nerve stimulation to treat inflammation. Based on template provided by Preferred Reporting Items for Systematic Reviews and Meta-Analyses (PRISMA) statement at http://prisma-statement.org. 
Table 1. Summary of the systematic literature review on antiinflammatory effects of VNS treatment in animal models and human trials.

\begin{tabular}{|c|c|c|c|c|c|c|c|c|c|c|c|}
\hline & Ref & Sex & VNS site & Design & $\mathrm{F}(\mathrm{Hz})$ & $A(V)$ & $\mathrm{I}(\mathrm{mA})$ & Pulse (ms) & Duration (min) & Effect & Persistence \\
\hline \multirow[t]{11}{*}{ Mice } & (11) & $\mathrm{F}$ & LCV & C & 20 & & 0.8 & 0.5 & 0.5 & $\downarrow$ & $A$ \\
\hline & $(12)$ & & LCV & C & 5 & 1 & & 2 & 20 (10 b/a LPS) & $\downarrow$ & $c^{\$ \$}$ \\
\hline & & M & & $A$ & 5 & 1 & & 2 & 2 (1 b/a LPS) & & \\
\hline & & & & & 5 & 1 & & 2 & 0.5 (5 a/ LPS) & & \\
\hline & & & & & 30 & 1 & & 0.5 & 0.5 (5 a/ LPS) & & \\
\hline & (13) & M & RCV & A & 1 & & 2 & $?$ & $10 \mathrm{~b} /$ & $\downarrow$ & A \\
\hline & (14) & & & & & & & & & & \\
\hline & (15) & M & $\mathrm{RCV}$ & $A$ & & & 2 & & Intermittently for 10 & $\downarrow$ & A \\
\hline & (16) & M & $\mathrm{RCV}$ & A & & & 2 & & 10 & $\downarrow$ & A \\
\hline & (17) & M & $\mathrm{RCV}$ & $A$ & & 5 & & & 15 or 20 & $\downarrow$ & A \\
\hline & (18) & M & LCV & C & 5 & & 0.05 & 1 & 10 & $\downarrow$ & $C^{\$ \$ \$}$ \\
\hline \multirow[t]{21}{*}{ Rats } & $(6)$ & $M$ & R/LCV & A & 1 & 5 & & 2 & 20 (10 b/ and $10 a / L P S)$ & $\downarrow$ & A \\
\hline & (19) & M & LCV & A & 2 & & 2 & 0.3 & 10 q45 b/ LPS & $\downarrow$ & A \\
\hline & $(8)$ & M & LCV & $A$ & 5 & 5 & & 2 & 3 & $=$ & A \\
\hline & (20) & M & LCV & A & 1 & 5 & & 2 & $20(10 \mathrm{~b} /$ and $10 \mathrm{a} / \mathrm{LPS})$ & $\downarrow$ & A \\
\hline & $(21)$ & $M \& F$ & LCV & C & 20 & & 0.25 & 500 & $\begin{array}{l}0.5 \text { q5 for } 3 \mathrm{~h} \text { per } \mathrm{d} \text { from } \\
\mathrm{d} 1 \text { to } \mathrm{d} 6\end{array}$ & $\downarrow$ & A \\
\hline & (22) & M & LCV & A & 1 & 5 & & 2 & 20 a/ LPS & $\downarrow$ & $A$ \\
\hline & $(23)$ & M & R heart & A & 5 & $2-6$ & & 1 & $15 \mathrm{~b} /$ and $120 / \mathrm{a}$ & $\downarrow$ & A \\
\hline & $(24)$ & M & $\mathrm{RCV}$ & $A$ & $8-10$ & 2.5 & & 0.5 & 40 & $\downarrow$ & A \\
\hline & $(25)$ & M & R/LCV & $A$ & 1 & 5 & & 2 & $15 \mathrm{~b} / \mathrm{and} \mathrm{a} / 6 \mathrm{~h}$ & $\downarrow$ & $C^{\$}$ \\
\hline & $(26)$ & M & R/LCV & A & 1 & 5 & & 2 & 20 a/ LPS & $\downarrow$ & A \\
\hline & $(27)$ & M & LCV & $A$ & 5 & 10 & & 2 & $20 \mathrm{a} /$ & $\downarrow$ & $\mathrm{C}$ \\
\hline & (28) & M & $\mathrm{RCV}$ & $A$ & 5 & 15 & & 2 & 20 & $\downarrow$ & A \\
\hline & (29) & M & $\mathrm{RCV}$ & $A$ & 1 & 5 & & 2 & $\begin{array}{l}\text { Either } 20 \text { starting } 5 \text { b/ LPS or } \\
2 \text { ( } 1 \mathrm{~b} / \text { and } 1 \mathrm{a} / \mathrm{LPS})\end{array}$ & $\downarrow$ & A \\
\hline & (30) & - & LCV & $A$ & 10 & & 0.0005 & $1 \mathrm{~s}$ & $0.3 \mathrm{~b} / 45 \mathrm{a} / \mathrm{LPS}$ & $\downarrow$ & A \\
\hline & (31) & M & $\mathrm{LCV}$ & $A$ & 2 & & 2 & 0.3 & $\begin{array}{l}10 \mathrm{q} 45, \text { starting right b/ LPS, until } \\
4.5 \mathrm{~h} \text { a/ LPS }\end{array}$ & $\downarrow$ & $A$ \\
\hline & (32) & M & $\mathrm{RCV}$ & $A$ & 20 & & 0.5 & 0.5 & 0.5 train q 5 for 30 & $\downarrow$ & C \\
\hline & (33) & - & LCV & A & 10 & & 0.5 & 1000 & $\begin{array}{l}0.02 \text { with } 500 \text { us bipolar stimul } \\
\text { q0.3 for 10; repeat } 10 \text { a/ LPS for } \\
45 \mathrm{~b} / \text { break of } 3 \mathrm{~h}\end{array}$ & $\downarrow$ & A \\
\hline & (34) & M & $\mathrm{RCV}$ & $A$ & 5 & 5 & & & 10 & $\downarrow^{\S}$ & C \\
\hline & (35) & $\mathrm{F}$ & LCV & C & 20 & & 1 & 10 & $\begin{array}{l}3 \mathrm{~h} \text { at same time every other day, } \\
\text { beginning } 1 \text { wk after operation } \\
\text { for } 12 \text { wks }\end{array}$ & $\downarrow$ & A \\
\hline & (36) & M & RCV\# & A & 25 & 7.2 & & 1 & 2 q10 for 60 & $\downarrow$ & A \\
\hline & (37) & M & $\mathrm{RCV}$ & $A$ & 10 & $1.0-7.0$ & N/A & 2 & $\mathrm{~N} / \mathrm{A}$ & $\downarrow$ & A \\
\hline Pigs & (38) & M & R/LCV & $A$ & 3 & & 1 & 0.2 & & $n / a$ & \\
\hline \multirow[t]{2}{*}{ Dogs } & (39) & $M$ and $F$ & RCV & C & 20 & & $0.75-2.5$ & 0.5 & $\begin{array}{l}14 \text { s on } 12 \text { s off continuously for } \\
8 \text { wks }\end{array}$ & $\downarrow$ & A \\
\hline & $(40)$ & $\mathrm{F}$ & RCV & $A$ & 1 or 2 & & $0.02-50$ & 0.3 & 0.006 & $n / a$ & \\
\hline
\end{tabular}


Table 1. Continued.

\begin{tabular}{|c|c|c|c|c|c|c|c|c|c|c|c|}
\hline \multirow[t]{5}{*}{ Humans } & $(41)$ & $M$ and $F$ & LCV & C & $1-145$ & - & $\begin{array}{l}0.25-4 \text { in } \\
0.25 \text { steps }\end{array}$ & $130-1000$ & $7-270 \mathrm{~s}$ in $30 \mathrm{~s}$ steps & $\downarrow$ & C \\
\hline & $(9)$ & $M$ and $F$ & LCV & C & 30 & & $0.75-1.75$ & 500 & 0.5 on $5 \mathrm{~s}$ off for 3 months & $=$ & $A$ \\
\hline & (10) & M & $\mathrm{LCV}^{*}$ & A & 20 & $0-10$ & & 1 & Continuously for 30 & $=$ & C \\
\hline & $(42)$ & $M$ and $F$ & LCV & $A^{* *}$ & 20 & & 1 & 0.5 & 0.5 & $\downarrow$ & A \\
\hline & & & & $C^{* * * \S \S}$ & 10 & & $0.25-2$ & 0.25 & 1 & $\downarrow$ & $C^{\S \S}$ \\
\hline
\end{tabular}

Abbreviations: F, frequency, Hz; A, amplitude, volt; I, intensity, milliampere; Pulse, duration of a VNS pulse; Duration, duration of VNS stimulation treatment; A, acute effect $<24 \mathrm{~h}$ (on inflammation); C, chronic $>24 \mathrm{~h}$ (on the experimental endpoint); $\downarrow$, R of inflammation due to VNS; =, no effect; RCV, right cervical vagus; LCV, left cervical vagus

${ }^{*}$ Catheter inserted in the left internal jugular vein at spinal level 5-C7, adjacent to the vagus nerve

${ }^{* *}$ Epilepsy cohort

${ }^{* * *}$ Rheumatoid arthritis cohort

"Skin overlying the right cervical vagus nerve

\$Improvement of renal function at three days, return to control at five days

${ }^{\S}$ Reduced (except in $\alpha 7 n A C h R$ knockout mice)

${ }^{\S}$ Acute design for epilepsy cohort; chronic design for rheumatoid arthritis cohort

${ }^{\$ \$} 48 \mathrm{~h}$ but not $72 \mathrm{~h}$

${ }^{\text {ss }}$ VNS $10 \mathrm{~min}$ b/ ischemia resulted in no protection; protective effects for at least two days

severity was also observed. All studies except two used VNS treatment before inducing inflammatory response rather than after, to mimic a clinical scenario.

\section{DISCUSSION}

We report the diverse VNS methodological profile reviewing the currently used VNS antiinflammatory strategies in animal studies and human clinical trials. While tolerance for VNS is good, apart from its successful use in refractory epilepsy in humans, its antiinflammatory effects are not supported by the two human studies included in this review $(9,10)$. Notably, as of July 10,2016 , a search for "vagus nerve stimulation AND antiinflammatory effects" on clinicaltrials. gov turned up 11 registered studies, four of them actively recruiting. Seventy studies are listed for "vagus nerve stimulation," excluding epilepsy-related research. This is a testament to the high interest and hopes this nonpharmacologic treatment modality elicits among various fields of medicine and the relevance of creating and maintaining a uniform reporting standard for VNS.

The discrepancy between the promising antiinflammatory effects of VNS in animal and human studies may be due to a lack of basic physiological understanding of the "vagus code," that is, how the efferent and afferent signals are encoded in the vagus nerve and how the information about the various stimuli is represented within the efferent and afferent vagal pathways. Evidence exists from VNS studies that frequency coding may be an approach by which to discretely stimulate its antiepileptic ( $25 \mathrm{~Hz}$ activating vagal afferents) or antiinflammatory ( $\sim 5 \mathrm{~Hz}$ activating vagal efferents) effects (43). Recently, as proof of principle, temporal patterning of VNS has been applied to selectively stimulate vagus nerve fibers, inducing bradycardia (44). A systematic effort to decode communication via the vagus nerve is needed to devise more hypothesis-driven VNS paradigms that are likely to lead to dedicated immune-modulatory effects in humans. This represents the promise and mission of bioelectronic medicine and the National Institutes of Health Brain Research through Advancing Innovative Neurotechnologies (BRAIN) initiative.

The first step should be a consistent reporting framework for VNS studies. We propose that such framework should include the variables presented in Table 1 of this review. The lack of consistency in reporting the VNS paradigms makes it difficult to validate and develop some of the pioneering work done in this field.

Another reason for the discouraging results in human studies is the male gender bias, which became apparent when reviewing the animal, mostly rodent, literature.

Furthermore, the myelinated part of the vagus nerve is phylogenetically more recent than the nonmyelinated; differences among species and during development exist as to the degree of myelination (45-47). Such functional anatomical differences should have an impact on VNS results.

Recent work in molecular genetics provides another rich dimension to the complexity of the vagus code: pre- and post-transcriptional and epigenetic modifications govern the bioavailability of acetylcholine, the carrier of vagus code within the central and peripheral nervous systems, as well as in the neuroimmunological synapse, via the species-specific variants of microRNA (miRNA or miR) and alternative splicing, ultimately resulting in the complex spatiotemporal landscape of acetylcholine esterase variants (48-51). 
Some of these miRNA, for example miR-608, are primate-specific. SNP variants in the miR-608 binding region modify miR-608-mediated suppression of acetylcholine esterase, and consequently activity of the autonomic and central nervous systems in humans (52-54). This explains our finding that rodent VNS models may not be good predictors of VNS treatment effects in humans.

Lastly, we found no studies on developing organisms, from perinatal to juvenile age, where putative salutary effects of VNS would be investigated, although CAP is active as early as in the late-gestation fetus $(55,56)$. There is a continued need to provide better treatments for sepsis, severe infection and cardiovascular compromise in early life $(55,57)$.

\section{CONCLUSION}

Overall, this review reveals the nascent stage in which the field of VNS treatment of inflammation finds itself 16 years since its inception (6). The results of the animal studies are very promising and call for a theoretical modeling of vagus code accounting for all levels of organization, from systems biology to systems physiology; a more systematic approach to experimental design and reporting; consideration of the gender effect on inflammation (58) developmental stages; and more diverse animal models (to better gauge the putative species diversity in the vagus code) to ultimately harness the salutary potential of this treatment modality. Such framework has the potential to lead to the development of truly personalized VNS regimens. Lastly, concerted and well-funded efforts are required to devise noninvasive alternatives to VNS to translate this treatment approach into widely used clinical experimentation, and eventually practice, to benefit patients.

\section{ACKNOWLEDGMENTS}

Supported by grants from the Canadian Institute of Health Research (MGF) and Fonds de la recherche en santé du Québec (MGF).

\section{DISCLOSURE}

The authors declare that they have no competing interests as defined by Bioelectronic Medicine, or other interests that might be perceived to influence the results and discussion reported in this paper.

\section{REFERENCES}

1. Patel KS, et al. (2013) Efficacy of vagus nerve stimulation as a treatment for medically intractable epilepsy in brain tumor patients. A case-controlled study using the VNS therapy Patient Outcome Registry. Seizure. 22(8): 627-33.

2. Elliott RE, et al. (2011) Efficacy of vagus nerve stimulation over time: review of 65 consecutive patients with treatment-resistant epilepsy treated with VNS >10 years. Epilepsy Behav. 20(3):478-83.

3. Uthman, BM. (2000) Vagus nerve stimulation for seizures. Arch. Med. Res. 31(3):300-03.

4. Bonaz B, et al. (2016) Chronic vagus nerve stimulation in Crohn's disease: a 6-month follow-up pilot study. Neurogastroenterol. Motil. 28(6):948-53.

5. Lim HD, et al. (2016) Anti-Inflammatory Effects of Acupuncture Stimulation via the Vagus Nerve. PLoS One. 11(3):e0151882.

6. Borovikova LV, et al. (2000) Vagus nerve stimulation attenuates the systemic inflammatory response to endotoxin. Nature. 405(6785):458-62.

7. Pavlov VA, Tracey KJ. (2015) Neural circuitry and immunity. Immunol. Res. 63(1-3):38-57.

8. Kox M, et al. (2012) Effects of vagus nerve stimulation and vagotomy on systemic and pulmonary inflammation in a two-hit model in rats. PLoS One. 7(4):e34431.

9. Barone L, et al. (2007) Effect of vagal nerve stimulation on systemic inflammation and cardiac autonomic function in patients with refractory epilepsy. Neuroimmunomodulation. 14(6):331-36.

10. Kox M, et al. (2015) Transvenous vagus nerve stimulation does not modulate the innate immune response during experimental human endotoxemia: a randomized controlled study. Arthritis Res. Ther. 17:150.

11. Krahl SE, et al. (1998) Locus coeruleus lesions suppress the seizure-attenuating effects of vagus nerve stimulation. Epilepsia. 39(7):709-14.

12. Huston JM, et al. (2007) Transcutaneous vagus nerve stimulation reduces serum high mobility group box 1 levels and improves survival in murine sepsis. Crit. Care Med. 35(12):2762-68.

13. Costantini TW, et al. (2010) Vagal nerve stimulation protects against burn-induced intestinal injury through activation of enteric glia cells. Am. J. Physiol. Gastrointest. Liver Physiol. 299(6):G1308-18.

14. Costantini TW, et al. (2010) Efferent vagal nerve stimulation attenuates gut barrier injury after burn: modulation of intestinal occludin expression. J. Trauma. 68(6):1349-54; discussion 1354-56.
15. Lopez NE, et al. (2012) Vagal nerve stimulation blocks peritoneal macrophage inflammatory responsiveness after severe burn injury. Shock. 38(3):294-300.

16. Bansal V, et al. (2012) Vagal stimulation modulates inflammation through a ghrelin mediated mechanism in traumatic brain injury. Inflammation. 35(1):214-20.

17. Vida G, et al. (2011) Alpha7-cholinergic receptor mediates vagal induction of splenic norepinephrine. J. Immunol. 186(7):4340-46.

18. Inoue $T$, et al. (2016) Vagus nerve stimulation mediates protection from kidney ischemiareperfusion injury through alpha7nAChR+ splenocytes. J. Clin. Invest. 126(5):1939-52.

19. Mihaylova S, et al. (2012) Effects of antiinflammatory vagus nerve stimulation on the cerebral microcirculation in endotoxinemic rats. J. Neuroinflammation. 9:183.

20. van Westerloo DJ, et al. (2006) Vagus nerve stimulation inhibits activation of coagulation and fibrinolysis during endotoxemia in rats. J. Thromb. Haemost. 4(9):1997-2002.

21. Sun P, et al. (2013) Involvement of MAPK/ NF-kappaB signaling in the activation of the cholinergic anti-inflammatory pathway in experimental colitis by chronic vagus nerve stimulation. PLoS One. 8(8):e69424.

22. Chen C, et al. (2013) Vagal efferent fiber stimulation ameliorates pulmonary microvascular endothelial cell injury by downregulating inflammatory responses. Inflammation. 36(6):1567-75.

23. Zhao M, et al. (2013) Vagal stimulation triggers peripheral vascular protection through the cholinergic anti-inflammatory pathway in a rat model of myocardial ischemia/reperfusion. Basic Res. Cardiol. 108(3):345.

24. Calvillo L, et al. (2011) Vagal stimulation, through its nicotinic action, limits infarct size and the inflammatory response to myocardial ischemia and reperfusion. J. Cardiovasc. Pharmacol. 58(5):500-07.

25. Hoeger S, et al. (2010) Modulation of brain dead induced inflammation by vagus nerve stimulation. Am. J. Transplant. 10(3):477-89.

26. Shi DG, et al. (2003) [Effects of efferent vagus nerve excitation on inflammatory response in heart tissue in rats with endotoxemia]. Zhongguo Wei Zhong Bing Ji Jiu Yi Xue. 15(1):26-28.

27. Yamakawa K, et al. (2013) Electrical vagus nerve stimulation attenuates systemic inflammation and improves survival in a rat heatstroke model. PLoS One. 8(2):e56728.

28. Boland C, et al. (2011) Electrical vagus nerve stimulation and nicotine effects in peritonitisinduced acute lung injury in rats. Inflammation. 34(1):29-35.

29. Schulte A, et al. (2014) Loss of vagal tone aggravates systemic inflammation and cardiac impairment in endotoxemic rats. J. Surg. Res. 188(2):480-88.

30. Garcia-Oscos F, et al. (2015) Vagal nerve stimulation blocks interleukin 6-dependent synaptic 
hyperexcitability induced by lipopolysaccharideinduced acute stress in the rodent prefrontal cortex. Brain Behav. Immun. 43:149-58.

31. Mihaylova S, et al. (2014) Effects of antiinflammatory vagus nerve stimulation in endotoxemic rats on blood and spleen lymphocyte subsets. Inflamm. Res. 63(8):683-90.

32. Jiang Y, et al. (2015) PPARgamma upregulation induced by vagus nerve stimulation exerts anti-inflammatory effect in cerebral ischemia/ reperfusion rats. Med. Sci. Monit. 21:268-75.

33. Garcia-Oscos F, et al. (2015) Activation of the antiinflammatory reflex blocks lipopolysaccharideinduced decrease in synaptic inhibition in the temporal cortex of the rat. J. Neurosci. Res. 93(6):859-65.

34. Morishita K, et al. (2015) Neuroenteric axis modulates the balance of regulatory $\mathrm{T}$ cells and T-helper 17 cells in the mesenteric lymph node following trauma/hemorrhagic shock. Am. J. Physiol. Gastrointest. Liver Physiol. 309(3):G202-08.

35. Li P, et al. (2016) Chronic vagus nerve stimulation attenuates vascular endothelial impairments and reduces the inflammatory profile via inhibition of the NF-kappaB signaling pathway in ovariectomized rats. Exp. Gerontol. 74:43-55.

36. Ay I, et al. Transcutaneous Cervical Vagus Nerve Stimulation Ameliorates Acute Ischemic Injury in Rats. Brain Stimul. 9(2):166-73.

37. Yi C, et al. (2016) Vagus nerve stimulation attenuates myocardial ischemia/reperfusion injury by inhibiting the expression of interleukin-17A. Exp. Ther. Med. 11(1):171-76.

38. Schneider R, et al. (2010) A new vagal anchor electrode for real-time monitoring of the recurrent laryngeal nerve. Am. J. Surg. 199(4):507-14.

39. Zhang Y, et al. (2009) Chronic vagus nerve stimulation improves autonomic control and attenuates systemic inflammation and heart failure progression in a canine high-rate pacing model. Circ. Heart Fail. 2(6):692-99.

40. Yoo PB, et al. (2013) High-resolution measurement of electrically-evoked vagus nerve activity in the anesthetized dog. J. Neural Eng. 10(2):026003.

41. Schachter SC, Saper BC. (1998) Vagus nerve stimulation. Epilepsia. 39(7):677-86.

42. Koopman FA, et al. (2016) Vagus nerve stimulation inhibits cytokine production and attenuates disease severity in rheumatoid arthritis. Proc. Natl. Acad. Sci. U.S.A. 113(29):8284-89.

43. Bonaz B, et al. (2013) Vagus nerve stimulation: from epilepsy to the cholinergic anti-inflammatory pathway. Neurogastroenterol. Motil. 25(3):208-21.

44. Yoo PB, et al. (2016) Modulation of heart rate by temporally patterned vagus nerve stimulation in the anesthetized dog. Physiological Reports. 4(2):e12689.

45. Brown SL, Brown RM, Penner LA. (2011) Moving Beyond Self-Interest: Perspectives from Evolutionary Biology, Neuroscience, and the Social Sciences. New York: Oxford University Press.
46. Agostoni E, et al. (1957) Functional and histological studies of the vagus nerve and its branches to the heart, lungs and abdominal viscera in the cat. J. Physiol. 135(1):182.

47. Sachis PN, et al. (1982) Myelination of the human vagus nerve from 24 weeks postconceptional age to adolescence. J. Neuropath. Exp. Neurol. 41(4):466-72.

48. Meydan C, Shenhar-Tsarfaty S, Soreq H. (2016) MicroRNA Regulators of Anxiety and Metabolic Disorders. Trends Mol. Med. 22(9):798-812.

49. Barbash S, Shifman S, Soreq H. (2014) Global coevolution of human microRNAs and their target genes. Mol. Biol. Evol. 31(5):1237-47.

50. Soreq H. (2015) Checks and balances on cholinergic signaling in brain and body function. Trends Neurosci. 38(7):448-58.

51. Nadorp B, Soreq H. (2014) Predicted overlapping microRNA regulators of acetylcholine packaging and degradation in neuroinflammation-related disorders. Front. Mol. Neurosci. 7:9.

52. Hanin G, et al. (2014) Competing targets of microRNA-608 affect anxiety and hypertension. Hum. Mol. Genet. 23(17):4569-80.

53. Lin $\mathrm{T}$, et al. (2016) Intensified vmPFC surveillance over PTSS under perturbed microRNA-608/ AChE interaction. Transl. Psychiatry. 6(5):e801.

54. Shenhar-Tsarfaty S, et al. (2016) Weakened cholinergic blockade of inflammation associates with diabetes-related depression. Mol. Med. 22:156-161.

55. Garzoni L, Faure C, Frasch MG. (2013) Fetal cholinergic anti-inflammatory pathway and necrotizing enterocolitis: the brain-gut connection begins in utero. Front. Integr. Neurosci. 7:57.

56. Frasch MG, et al. (2016) Decreased neuroinflammation correlates to higher vagus nerve activity fluctuations in near-term ovine fetuses: a case for the afferent cholinergic anti-inflammatory pathway? J. Neuroinflammation. 13(1):103.

57. Shane AL, Stoll BJ. (2014) Neonatal sepsis: progress towards improved outcomes. J. Infect. 68(Suppl 1):S24-32.

58. Mabley JG, et al. (2005) Gender differences in the endotoxin-induced inflammatory and vascular responses: potential role of poly(ADP-ribose) polymerase activation. J. Pharmacol. Exp. Ther. 315(2):812-20.

Cite this article as: Kwan H, et al. (2016) Vagus nerve stimulation for treatment of inflammation: Systematic review of animal models and clinical studies. Bioelectron. Med. 3:1-6. 